\title{
El inconsciente y el subconsciente
}

Autor: Macarena Márquez Gordillo, Alejandro Espina Díaz y José Manuel Toronjo Martín

Afiliación Alumnado de la asignatura "La educación en valores y la práctica de actividad física en colectivos de desigualdad. Grado de Ciencias de la Actividad Física y el Deporte. Universidad de Huelva.

Email:: marquez4157@gmail.com; alejandroespinadiaz@gmail.com; 


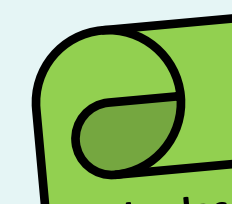

En la clase anterior

pág. 2

Pregunta del día ¿Qué dice la ciencia? pág. 3

El subconsciente y el inconsciente..........pág. 5

Aplicación a la actividad física. pág. 6

Para saber más. pág. 7-8

Artículos científicos

Libros

Comentarios de prensa

pág. 9

On-line

Programa de Intervención

Actividades.

Frase del día

Mapa conceptual.

Pasatiempos

Conclusiones

Bibliografía.

pág. 3-4

6

pág. 10 pág. 11 .pág. 12 pág. 13 pág. 14 pág. 15

pág. 15-16 


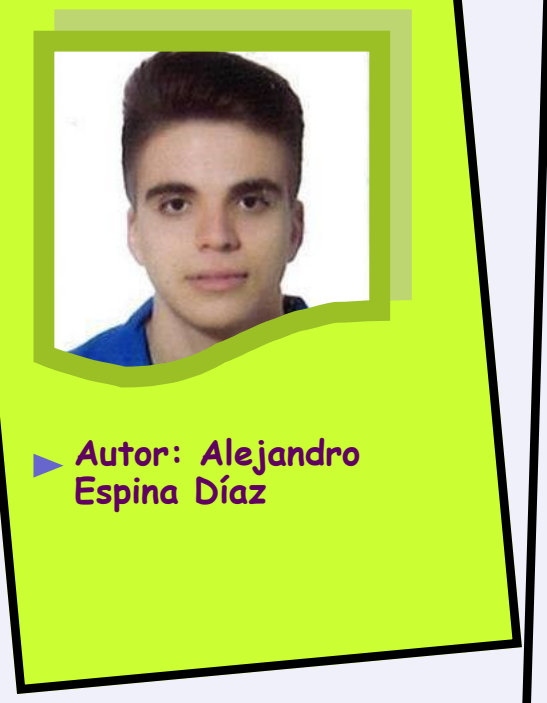

Sáenz-López, P. (2014). Apuntes inéditos de la asignatura "La educación en valores y la práctica de actividad física en
Realizó un resumen de la clase anterior, la cual comenzó con la visualización de dos vídeos, en el primer vídeo se explicaba que cuando un jugador va a lanzar un penalti recae mucha presión sobre él y pierde capacidad en la realización de los movimientos; $y$ en el segundo que un hombre con esclerosis gracias a realizar entrenamientos y competiciones de triatlón mejoró mucho su enfermedad, diciendo que éste deporte había sido su medicamento y curación.

Posteriormente, hicimos un juego en el que se le asignó un número a cada alumno de la clase y tenían que buscar al alumno que tuviese el mismo número asignado para contarse alguna experiencia en la que las emociones hayan influido en la actividad física.

En materia de clase, el tema expuesto trataba sobre la Inteligencia, que es la capacidad de resolver problemas.
Uno de sus tipos era la Inteligencia Emocional, que es la capacidad de resolver problemas emocionales internos; ésta a su vez se divide en Autoconocimiento, Autocontrol, Automotivación y Empatía. Después, se expuso los beneficios de la Inteligencia Emocional.

A continuación, realizamos una actividad con la pareja del juego anterior en la que teníamos que elegir una emoción y decir que influencia tiene en el contexto deportivo y qué podemos hacer como profesionales para gestionarla y acabar controlándola.

Como conclusiones de esta clase se dijo que de nosotros depende hacer las cosas con motivación o amargados, ya que nosotros somos nuestros propios guías; y que deberíamos centrarnos en cómo evitar las emociones negativas para convertirlas en positivas.
El profesor dedicó unos minutos a la actividad realizada por pareja en la clase anterior, en la que la emoción que más se eligió fue

\section{Sáenz-} López, P. (2014). Apuntes inéditos de la asignatura "La educación en a ira, siendo la autoestima eedback positivos) el remedio principal para solucionar los conflictos. También, comentamos el miedo cuyo antídoto principal es la confianza.

Posteriormente, se habló de la vergüienza comentando que en las actividades individuales se pasa más vergüenza que en las colectivas, por lo que se tienen que proponer actividades en la que los alumnos se sientan cómodos para que se desinhiban.

En cuanto a la culpa, se dijo que es una de las emociones más perjudiciales al tener muy pocos puntos positivos, por lo que debemos intentar cambiar la culpa por la responsabilidad.

Por último, el profesor aclaró que las charlas racionales no llegan al cerebro emocional y que un vídeo impacta mucho más, por ejemplo poner un vídeo sobre los accidentes de coches impacta mucho más que asistir a una charla.

\section{valores y la}




\section{LA PREGUNTA DEL DÍA}

\section{¿Es importante el inconsciente?}

\section{QUÉ DICE LA CIENCIA}

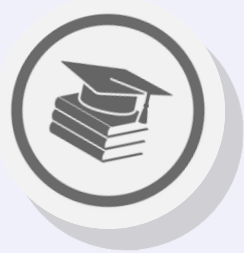

Alejandro nos presentó el artículo "Educación en la autoestima" el cual trata sobre la importancia e influencia de la autoestima en la estabilidad emocional de las personas. Las autoras distinguen tres ámbitos de intervención: familiar, educativo y social.

Según las autoras, la autoestima es fundamental para alcanzar la madurez personal y ello debe ser considerado por cualquier educador/a como un aspecto fundamental para desarrollar la personalidad de los alumnos. Desde el ámbito deportivo no debemos valorar los progresos de los alumnos en relación de los éxitos deportivos obtenidos frente al de los compañeros, sino reconocer las capacidades de cada uno de ellos y el progreso en función de sus capacidades.

Ser comprendido tiene un gran valor para la persona con baja autoestima, por ello uno de los mayores compromisos de un centro educativo debe ser facilitarles vivencias satisfactorias.

La falta de autoestima puede derivar en una inadaptación del sujeto. El concepto de la autoestima es un sentimiento de estima hacia uno mismo.
Como comentario personal, Alejandro destacó varias partes del estudio; "una palabra agradable puede ser el medio de salvar el sentido de una vida que de otro modo puede trastornarse para siempre". Cualquier educador al estar a cargo de un equipo de jóvenes debe proponerse como objetivo desarrollar la autoestima en cada uno de sus jugadores.

En un segundo estudio, Alejandro nos muestra el artículo "La actividad física mejora el aprendizaje y el rendimiento escolar", donde manifiesta que la práctica deportiva permite al niño/a ganar habilidades mediante el aprendizaje y las nuevas capacidades adquiridas que contribuyen a fortalecer su autoestima.

Estrategias para fomentar la autoestima en un equipo:

- Diseñar tareas no demasiado complejas con el fin de que todos obtengan éxito.

-Potenciar conductas o gestos deportivos a través de refuerzos positivos.

-Evitar comparaciones, clasificaciones y distinciones entre los compañeros.

-Intentar que todos participen, rotar el papel del capitán y que realicen la misma duración en el juego.

-En el diseño de sesiones de tareas de colaboración-oposición equilibrar los equipos.

- Crear un buen clima de trabajo.

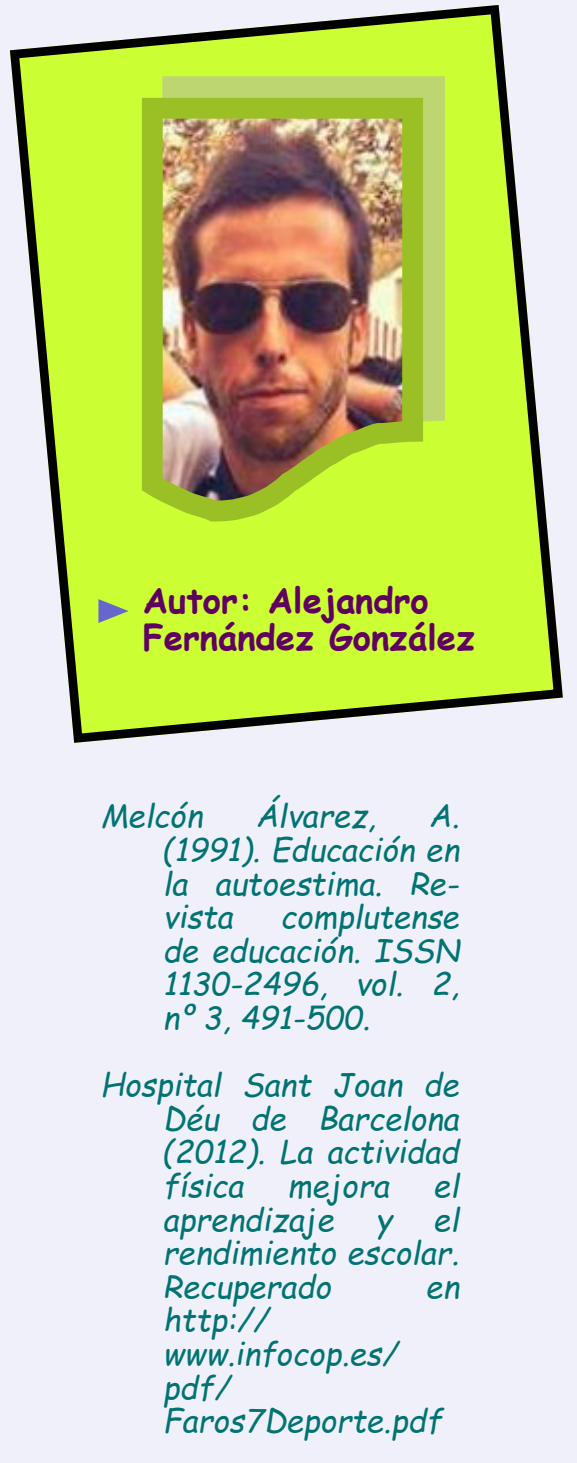




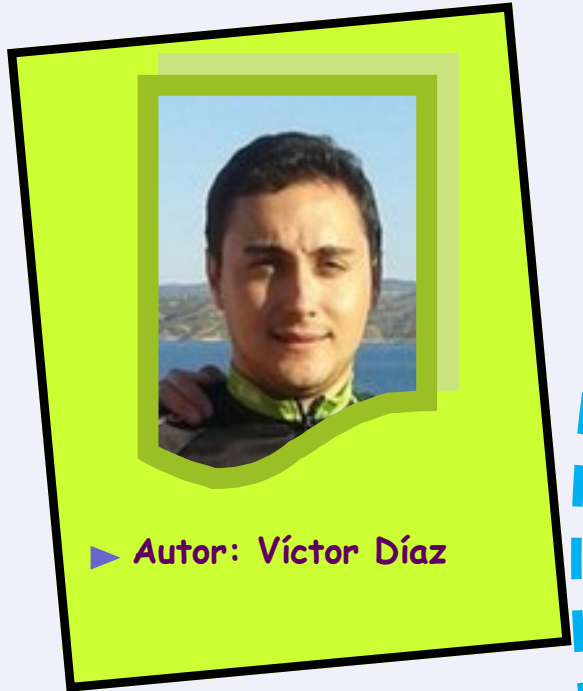

Moreno, J.A. et al. (2006). Importancia de la práctica físico -deportiva y del género en el auto concepto físico de los 9 a los 23 años.

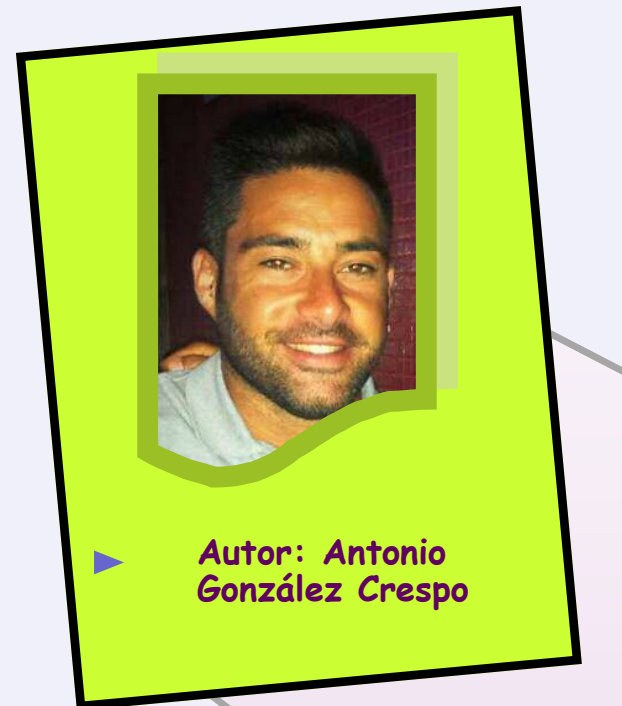

Vázquez, Piatti, M. (2008). El autoconocimiento, la base de la autoestima. $A B C$. Recuperado en: http:// www.abc.com.py/ apticulos/elautoconocimiento-la -base-de-laautoestima1059212.htm/
¿Qué es la autoestima?

Es la actitud hacia sí mismo que depende de cuatro factores: auto concepto, auto confianza, rol e imagen. Es posible construir una autoestima sâna en cualquier etapa del desarrollo humano. Pero sería ideal que esta se desarrollara desde la infancia para que en la edad adulta sea lexitosa.

\section{Importancia de la autoestima}

"La autoestima positiva opera en el sistema inmunológico de la conciencia, otorgando resistencia, fuerza y la capacidad de regeneración. Cuando la autoestima es baja, nuestra capacidad de enfrentar las adversidades de la vida bajan. Nos caemos frente a las adversidades $y$ nuestro sentido de propio valor disminuye. Nos dejamos influenciar por el deseo de evitar el dolor, en vez de experimentar alegría. Todo lo negativo tiene más influencia en nosotros, en vez de lo positivo." Nathaniel Branden
El propósito de este estudio descriptivo mediante encuesta es comprobar los efectos de la edad, el género y la práctica físico-deportiva en el auto concepto físico en estudiantes de 9 a 23 años. La muestra está compuesta por 2.332 alumnos a los que se les pasó el Physical Self 1 -concept Questionary (PSQ).

Como conclusión, este estudio muestra la relevancia que la edad, el género y la práctica físico-deportiva extraescolar parecen tener sobre la elaboración del auto concepto físico.

Este estudio presenta la relación del auto concepto físico con diferentes variables sociodemográficas como la edad, el género y la práctica físico-deportiva extraescolar en estudiantes de distinto nivel educativo. Los resultados generales muestran que existe influencia de las variables de edad, género y práctica físico-deportiva en el auto concepto físico.. Mayor en hombres que en mujeres. A mayor horas de practica mejor auto concepto físico y autoestima.. La práctíca de actividad física mejora el auto concepto en todas las edades.
Antonio presentó el artículo de opinión "El autoconocimiento, la base de la autoestima". En el artículo se explica que el autoconocimiento es resultado de un proceso reflexivo mediante el cual la persona adquiere noción de su persona, de sus cualidades y características. El autorrespeto. Algunas personas desean que los demás la respeten, que les den su lugar, pero tal vez, sin darse cuenta, ellas mismas no creen que sean dignas o merecedoras de ese respeto. El respeto por uno mismo se basa en el conocimiento y aceptación de las propias limitaciones y posibilidades. El conocimiento y el autorrespeto conducen al propio control. Uno mismo es capaz de controlar sus impulsos, de postergar ciertas acciones para mejores ocasiones, es capaz de adaptarse a diferentes situaciones y lograr así ser mejor persona. No se trata de dejarse llevar o hacer todo lo que los demás dicen.
Por ejemplo, una joven que sabe que al tomar bebidas alcohólicas en una fiesta hace que ella hable de más, se ponga agresiva o llorona, que al día siguiente le duela la cabeza, tenga problemas con sus padres, su novio o en el colegio y luego se arrepienta, se sienta mal y, aun así toma, significa, que no se autocontrola, que no tiene mucho respeto por sí misma. Cuando se consigue el conocimiento, el respeto y control de uno mismo, con seguridad se obtiene una sana autoestima o autoestima positiva.

Puntuación del profesor: "es interesante los artículos de opinión de personas potentes, pero detrás de ellos existen investigaciones, experiencias, estudios,... Aunque sacar un artículo científico también nos da la veracidad para acompañar a lo que estamos diciendo" 

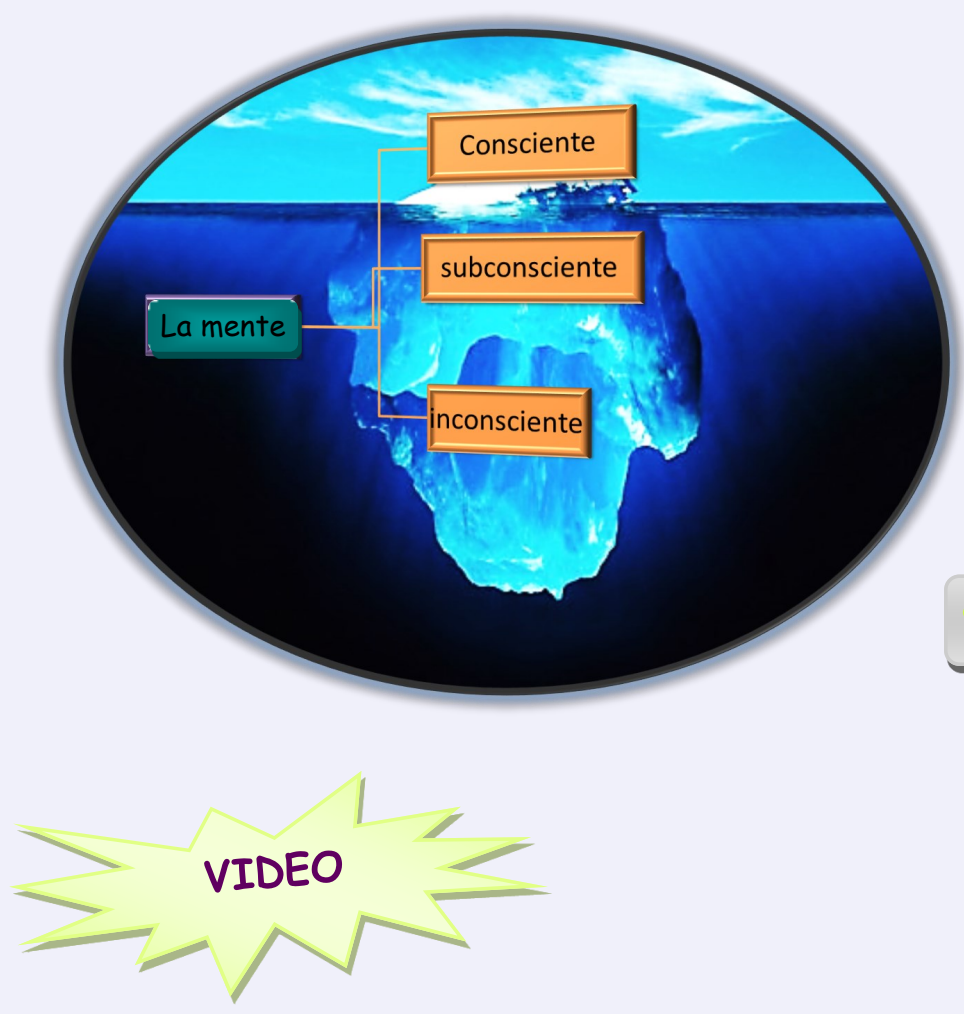

¿Qué es el subconsciente?

http://www.youtube.com/watch?v=H9DjChVsTbY

El inconsciente es el conjunto de todos los pensamientos y sentimientos que se crean en nuestra mente a los que no podemos acceder. Nos ayuda a entender el mundo que nos rodea haciéndonos tomar las decisiones que mejor nos convengan, es como una especie de piloto automático en el que podemos confiar, un GPS emocional, una guía de conducta que seguimos sin darnos cuenta.

La vida sin el inconsciente sería casi imposible, ya que lo encontramos en nuestros actos más cotidianos desde los automatismos que nos permiten levantarnos por las mañanas, vestirnos y caminar hasta los sentimientos más profundos que nos impulsan a defender a nuestros seres queridos.

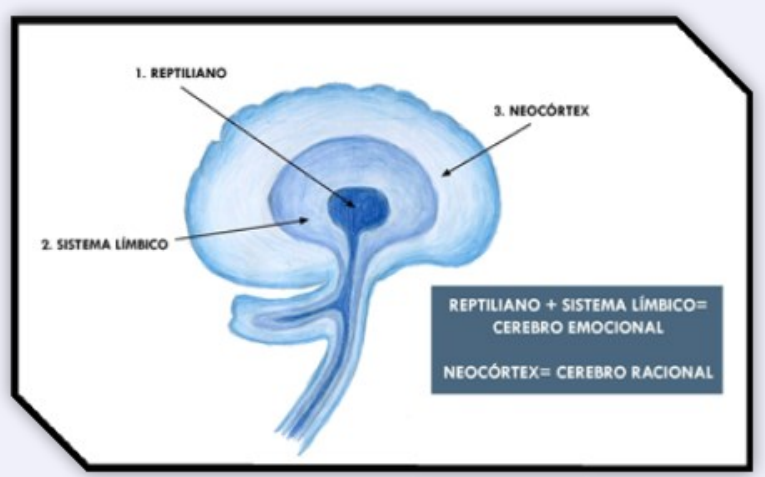


¿Cómo un futbolista ante tal cantidad de estímulos tanto intrínsecos como extrínsecos que se encuentra en una sola acción de un partido es capaz de ponerle solución de forma tan rápida y eficaz?
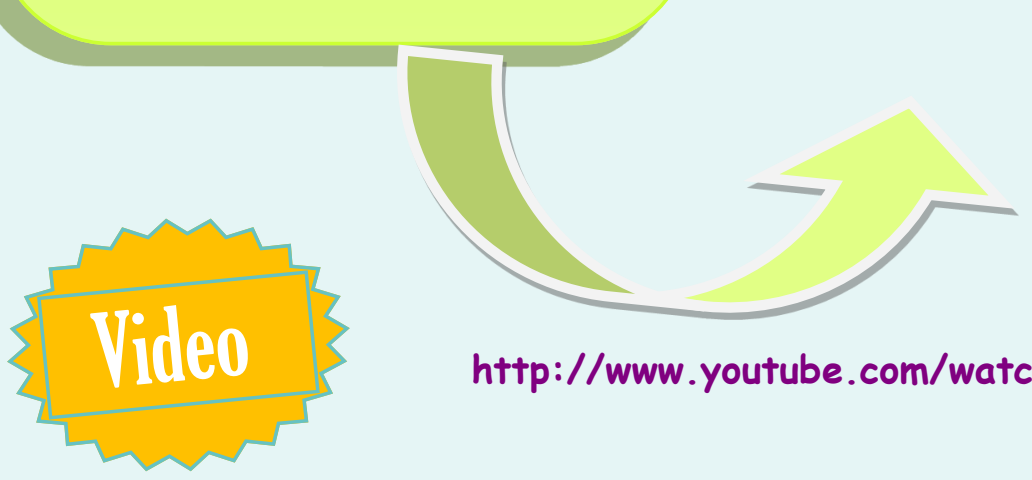

$h+t p: / / w w w$. youtube .com/watch?v=TyrISGU+6cy
Solución: La práctica y repetición de situaciones reales de juego, en las que el jugador experimenta problemas similares a los encontrados durante la competición, enriquece el bagaje emotivo experimental de éste, aumentando así el número de recursos a los que nuestro inconsciente podrá echar mano cuando se encuentre en apuros"

Un deportista de élite recibe miles de estímulos a lo largo de una jugada y la mayor parte son recibidos por el inconsciente. Por ejemplo, si se le preguntara a Leo Messi todo lo que ha hecho en una jugada, él no sabría contestarte exactamente debido a que la mayor parte de las cosas las ha realizado de forma inconsciente. Por eso, mientras más experiencia se tenga es mejor, por lo que un jugador experimentado es mejor que un jugador principiante.

(Álvaro Linaza, 2014)
Ningún ordenador del mundo dominará jamás el mar ya que cambia constantemente y cada ola es diferente a la anterior. Lo que mantiene a los surferos a flote es un maravilloso truco de nuestra mente, la intuición humana. Estos no piensan lo que tienen que hacer cuando se ven rodeados de agua, ya que sus cerebros ponen en marcha tareas muy rápidas calculando que ocurrirá después y cómo deben reaccionar sus músculos ya que su mente ha almacenado sin que lo sepan todas las olas que han vivido a lo largo de sus vidas, teniendo un sentimiento para cada ola.
Un surfista anticipa como va a ser la siguiente ola y como debe adaptarse, esto en el modo consciente no seríamos capaces de lidiarlo debido a que el mar es un entorno complicado e impredecible.

Tanto al enamorarse o jugar al baloncesto siempre aprendemos de la experiencia, si tuviéramos que coordinar conscientemente todos los movimientos dejaríamos de funcionar rápidamente, porque pensar constantemente quema mucha energía. Una parte del cerebro calcula las trayectorias del balón y de los jugadores, otra planifica todos los movimientos enviando comando a través de las áreas motoras primarias para llevarlos a la médula espinal y esta a las fibras musculares.
El control consciente es cansado y dado a sufrir accidentes ya que nos ralentiza. Cuando el cerebro actúa sin nosotros el rendimiento máximo se convierte en un juego de niños.

Lanzamos y recogemos balones fácilmente y no pensamos en ello, porque no sabemos exactamente lo que hace nuestros dedos al atraparlo y es mejor no pensar en ello porque su cerebro podría contradecirse a sí mismo, ralentizándose y cometiendo errores. Funcionar inconscientemente significa funcionar eficazmente y adaptarse a cambios repentinos es lo último para nuestra mente

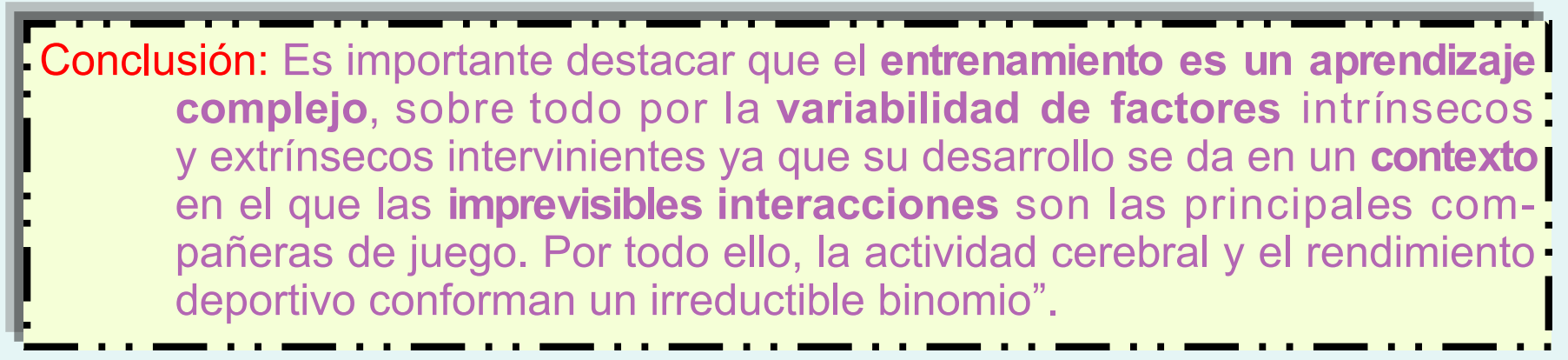

Espina, A., Márquez, M., Toronjo, J.(2014). Comentario sobre el video "El poder de la men- 


\section{El Inconsciente: Una Mirada sobre su Historia y sus Retos Actuales}

Las variadas caracterizaciones con las ha estado asociado lo inconsciente obligan a la creación de un esquema organizador de las discusiones presentes incluso antes del nacimiento de la psicología experimental.

Estos debates los hemos distribuido en tres ejes de análisis, el primero que corresponde a las caracterizaciones positivonegativo, el segundo que refiere a la mirada de lo inconsciente como activo-pasivo y el tercero que estudia el problema de la subjetividad asociado a la actividad inconsciente. El objetivc es mostrar cómo estas primeras consideraciones vuelven a tomar fuerza en los debates actuales al respecto del papel del inconsciente en el estudio de la mente humana y su relación con la consciencia.

En la parte final señalamos algunos de los aspectos que debería tomar en consideración la investigación actual sobre lo inconsciente y posibles retos en esta interesante tarea.

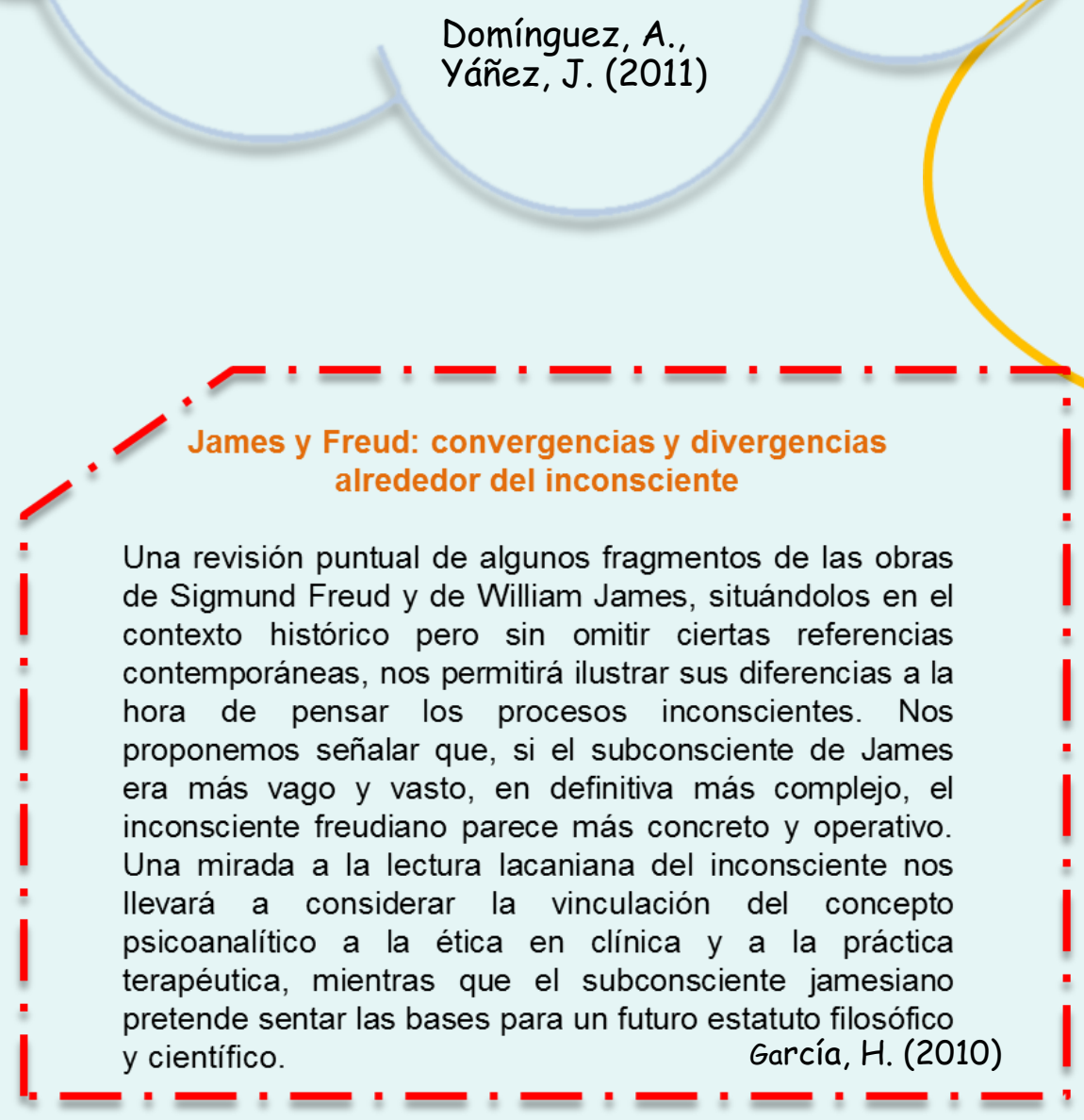

\section{El miedo al éxito}

El presente artículo aborda la manera en que el miedo al éxito sabotea a los deportistas en sus carreras deportivas en general, y específicamente en los momentos importantes y/o decisivos para lograr sus objetivos y el éxito en si. disciplina. Nieri, D. (1992) 


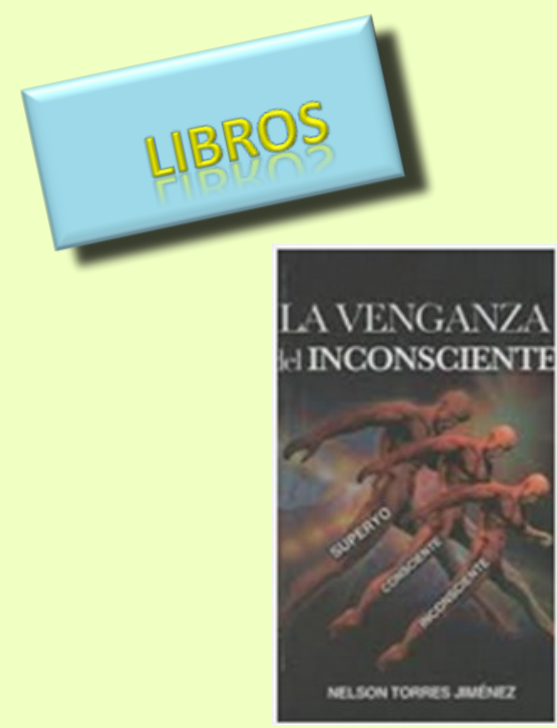

En este libro se presentan inicialmente algunas diferencias entre los clásicos SUPERYO, YO, y el INCONSCIENTE. Se presentan algunas enfermedades causadas por la Mente (en las Venganzas), para luego explicar el Pensamiento Caótico Versus el Pensamiento Co-Activo, y finalizar con un intercambio epistolar entre el YO, el SUPER YO y el INCONSCIENTE.

\begin{abstract}
Wl poder de tu mente subconsciente: usando el poder de tu mente puedes alcanzar una prosperidad, una felicidad y una paz mental sin límites (Murphy, 2008)

Este libro es una edición autorizada, revisada y aumentada por el Dr. Murphy, y presenta y explica diversas técnicas de proyección mental que nos permiten salvar aquellos obstáculos subconscientes que impiden la consecución del éxito que tanto deseamos y merecemos. La obra del Dr. Murphy se vale de ejemplos tomados de la vida real para enseñarnos la forma de liberar extraordinarios poderes mentales que, además de aumentar la confianza en nosotros mismos, crean relaciones armoniosas, nos facilitan el éxito profesional, acrecientan nuestras riquezas, suprimen miedos y fobias, hacen desaparecer los malos hábitos, realizan curaciones y propician nuestra felicidad y bienestar general.
\end{abstract}
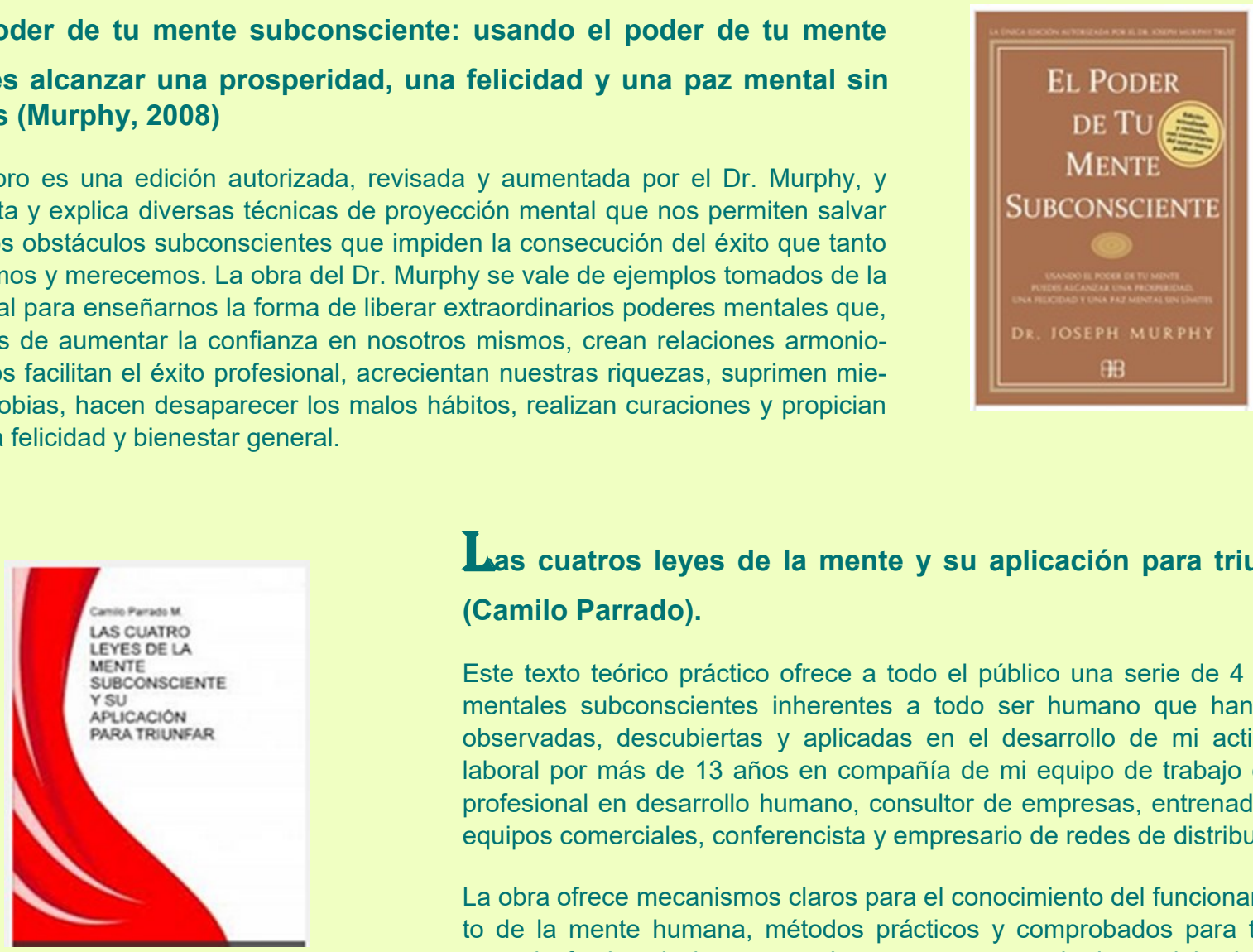

Las cuatros leyes de la mente y su aplicación para triunfar (Camilo Parrado).

Este texto teórico práctico ofrece a todo el público una serie de 4 leyes mentales subconscientes inherentes a todo ser humano que han sido observadas, descubiertas y aplicadas en el desarrollo de mi actividad laboral por más de 13 años en compañía de mi equipo de trabajo como profesional en desarrollo humano, consultor de empresas, entrenador de equipos comerciales, conferencista y empresario de redes de distribución.

La obra ofrece mecanismos claros para el conocimiento del funcionamiento de la mente humana, métodos prácticos y comprobados para tomar control efectivo de los pensamientos y como resultado modelar las circunstancias personales hacia una vida mejor.

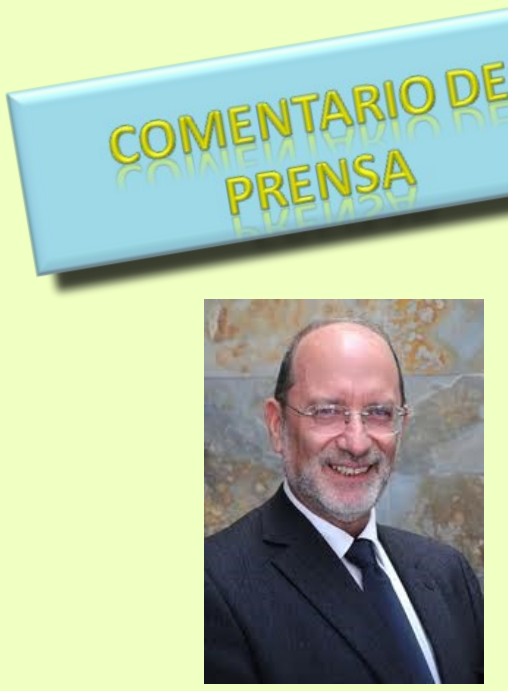

Autor: Henning

Jensen Pennington

\section{Reflexiones sobre el inconsciente y la historia en Sigmund Freud}

El autor estudia el lugar que ocupa el psicolamarckismo en la teoría de Sigmund Freud; es decir, su tesis acerca de la transmisión filogenética de las experiencias históricas de la especie humana. El artículo desarrolla tres hipótesis básicas: 1- el psicolamarckismo de Freud pretende brindar coherencia a la teoría psicoanalítica al establecer un vínculo entre dos etapas diferentes de su desarrollo; 2 - estas nociones aspiran a crear un puente entre la psicología individual y la colectiva y 3 - enuncian una teoría de la violencia social.

Jensen, $\mathrm{H}$. 


\title{
ON-LINE
}

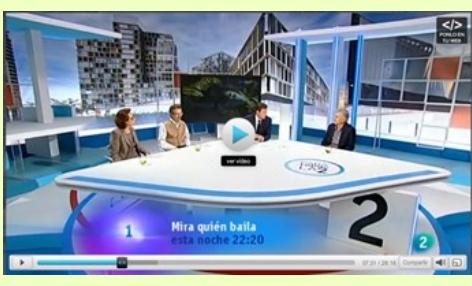

\section{Para Todos La 2 - Debate - El inconsciente}

\author{
http://www.rtve.es/alacarta/videos/para-todos-la-2/para-todos-2-debate-
} inconsciente/2351589/

El inconsciente es la parte de nuestra personalidad que guarda bajo llave recuerdos o deseos; o, desde otro punto de vista, los comportamientos que alguien desarrolla sin darse cuenta y, en general, sin quererlo. Coloquio entre Regina Bayo-Borràs, psicóloga clínica y psicoanalista, presidenta de la Sección de Psicoterapia Psicoanalítica de la Federación Española de Asociaciones de Psicoterapeutas; Álex Santos, médico e hipnoterapeuta; y Carles Ventura, Psicólogo Clínico y Presidente de la Sociedad Española de Psicología Analítica

\section{Redes - Las decisiones son inconscientes}

http://www.rtve.es/alacarta/videos/redes/redes-decisiones-soninconscientes/1016325/

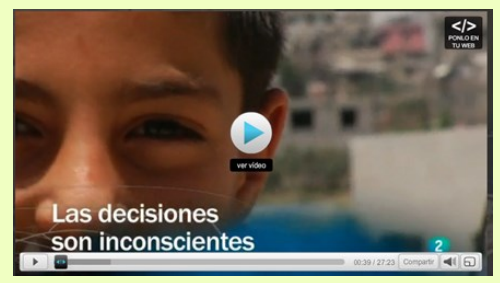

¿Somos libres cuando decidimos? ¿Qué margen tiene el libre albedrío ahora que la neurociencia desvela cada vez más el gran poder del inconsciente? Ya hay máquinas para ver cómo decide el cerebro y los primeros resultados muestran que, antes de entrar en la consciencia, muchas decisiones ya están tomadas por complejas redes cerebrales. Desde Berlín, John-Dylan Haynes nos habla en Redes, de sus experimentos para descifrar la actividad cerebral que se esconde detrás de nuestros estado mentales.

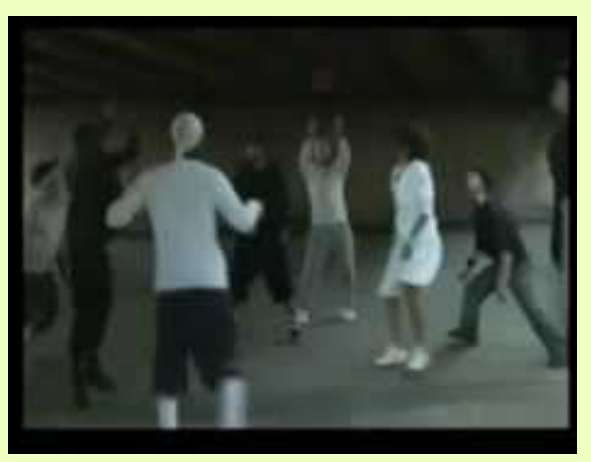

\section{Test de atención selectiva}

\section{https://www.youtube.com/watch?v=t4eaTx7bCew}

Es un vídeo de atención selectiva en el que hay dos equipos, uno con ropa blanca y otro negra. En este hay que contar los pases del equipo blanco y para ello tienes que centrar toda tu atención en ello. Durante el video se mete alguien con un disfraz de oso en la escena y al estar tan atentos en la cuenta de los pases, no nos damos cuenta de su presencia.

\section{Así decide el cerebro}

\section{http://www.youtube.com/watch?v=Obq6aZXxBhs}

Todo acto motor voluntario es involuntariamente iniciado en nuestro cerebro. Esto lo sabíamos: antes de ser conscientes de mover el dedo ya podemos ver en el cerebro que se va a producir un movimiento. Lo que no sabíamos es que cada decisión que tomamos, cada acto consciente es inconscientemente elaborado por nuestro cerebro. ¿Qué determina nuestras acciones voluntarias?

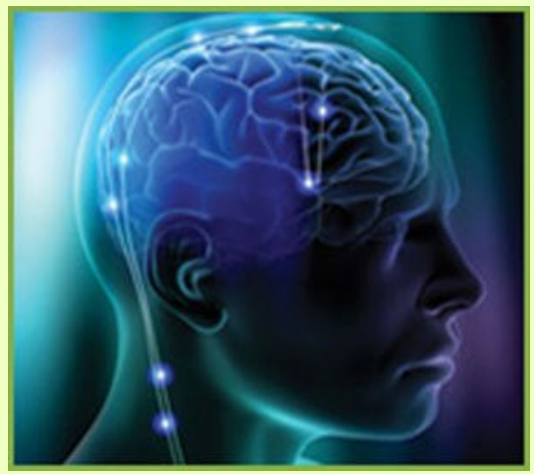




\section{ATENGION-CONGENTRACION COMO ENTRENAMIENTO PARA ZAME- JORA DEL RENDIMIENTO DEPOKTIVO EN JUGADORES PIKOFRSIONAZES

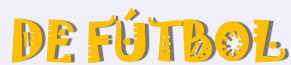

La investigación está dirigida a futbolistas profesionales y busca la mejora del rendimiento y la salud del deportista, tanto a nivel deportivo como personal. En palabras del propio Martens (1987, p. 16) «nuestra finalidad es ayudar a los deportistas a mejorar sus habilidades psicológicas para poder hacer frente a las presiones asociadas a la competición de alto nivel»; en este caso, centrado en la mejora de la capacidad atencional del futbolista, como medio de su mejora deportiva.

El presente trabajo de investigación con futbolistas profesionales tiene un claro referente cognitivo, incorporando también aspectos sociales, al intentar conseguir instaurar estrategias de mejora de la capacidad atencional en este grupo de deportistas de alta competición. Un tema muy interesante en el ámbito deportivo del fútbol es la atención-concentración del jugador. Parece ser que la concentración juega un papel fundamental en la competición, condicionando muy directamente aspectos tan relevantes como la precisión o la velocidad de ejecución motora. La atención se define como la capacidad cognitiva que permite seleccionar la modalidad de información deseada o requerida en un momento determinado y podríamos considerarla "como una habilidad que se aprende y mejora con la práctica, al igual que cualquier otra habilidad verbal o motora» (Oña, 1994, p. 186).

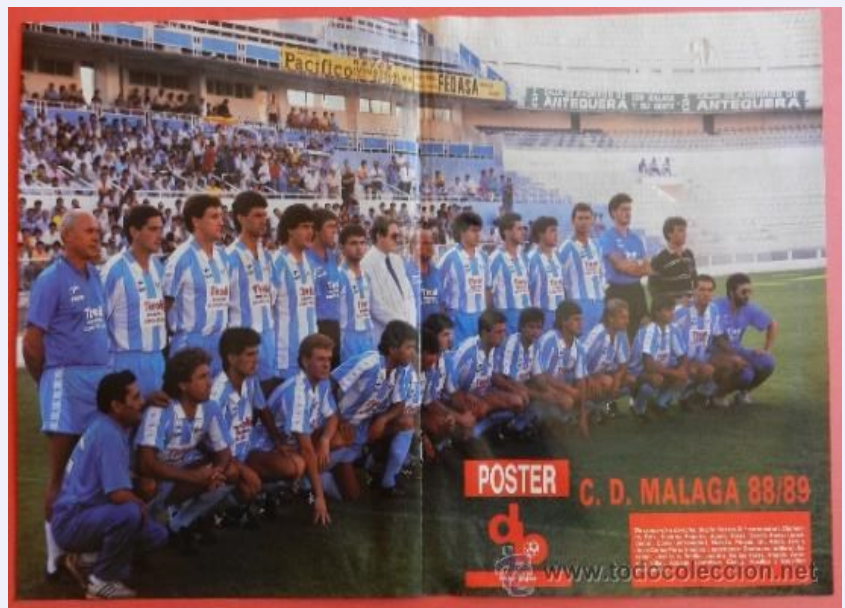

C. D.. Máallagat

\section{Discusión}

Los procedimientos de práctica atencional se basan por lo general en ejercicios mentales, no habiéndose integrado apenas el ensayo atencional dentro de la metodología tradicional de entrenamiento por parte del preparador del equipo. Será preciso, pues, realizar programas de entrenamiento adaptado específicamente al deporte de fútbol, en el que se estructuren ejercicios que ejerciten a los futbolistas en el desarrollo de estilos atencionales requeridos para este tipo de deporte, trabajando la atención en deporte como algo inherente al propio entrenamiento deportivo, en situación real, junto al entrenamiento físico, técnico y táctico, coordinados con el equipo técnico.

La dificultad se halla en la gran diferencia existente entre trabajar la atención desde un contexto de laboratorio y el trabajo en situación de entrenamiento real, llegando a poder demostrar que se da una mejora efectiva en el desarrollo del juego (rendimiento), y no solo en los aspectos técnicostácticos tal y como sucede en otros estudios.

La secuencia de las sesiones de trabajo puede ser otro aspecto a tener en cuenta. Su periodicidad semanal (a veces quincenal) prolonga excesivamente la intervención, dejando muy espaciados los momentos de aprendizaje, por lo que quizás el futbolista tenga ciertas dificultades a la hora de poner en práctica lo aprendido.

Por lo que respecta a la valoración del programa por parte de los futbolista, ésta ha resultado bastante positiva, destacando de manera especial, por un lado, el hecho de que la gran mayoría considere beneficiosa la preparación psicológica de cara a la práctica deportiva y, por otro, su gran utilidad con vistas a su mundo profesional. Los futbolistas aceptan un programa de estas características, les parece interesante y hasta necesario, constatando su eficacia en función de la extensión a todos los componentes del equipo. 


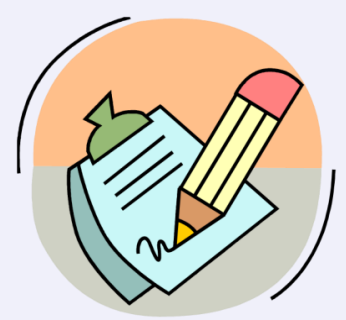

\section{- Gatillos emocionales}

Esta actividad consistió en que cada uno identificase su gatillos emocionales, es decir, algún miedo o sentimiento negativo que quedó guardado, latente, y que es reactivado o despertado apenas sucede una situación semejante en el presente. Cambia la época, los personajes... pero la reacción siempre es la misma. Por ejemplo las cosas que nos dan coraje nos siguen dando coraje siempre que se repitan. Debemos de concienciarnos para poder superarlos.

- ¿Qué parte de tu personalidad crees que es heredada y cual está influenciada por el entorno?. ¿Qué cambiarías y qué te gusta de ella?

Esta actividad consiste en tener que decir qué partes de tu personalidad crees que has heredado de tu familia y cual has adquirido debido al entorno que te rodea, teniendo que comentar qué es lo que te gusta de tu personalidad y qué te gustaría cambiar de ella.

- La ley del espejo

$\underline{h t t p: / / m e j o r c o n s a l u d . c o m / d e s c u b r e-l a-l e y-d e l-e s p e j o-p a r a-s e r-f e l i z / ~}$

Elegir una de esas personas (te molesta algo, enfadado, etc.) hacer una lista con cosas que nos gustaría agradecerle y otra exponiendo cosas por las que nos gustaría pedirle perdón. Nos ponemos en contacto con esa persona (personalmente, por teléfono o por carta), le daremos las gracias por todos los motivos apuntados en la lista, y le pediremos perdón por todas las cosas de la segunda lista.

Resultado: Este ejercicio anterior puede parecer una locura, puesto que muchas personas son demasiado orgullosas para llevarlo a cabo, o bien piensan que tendría que ser al revés, que son ellos los que deberían recibir los agradecimientos y dar el perdón. Pero el resultado suele ser sorprendente, y esas personas que jamás se esperarían un mensaje así suelen reaccionar de manera muy positiva y emocional.

- ¿Diga los tres últimos dígitos de tu número de teléfono?

¿Cuándo nació Genghis Khan?

48

843

1162

1354

Seguramente elegiste la respuesta B de forma inconsciente

Sáenz-López, P. (2014). Apuntes inéditos de la asignatura "La educación en valores y la práctica de actividad física en colectivos en situación de desigualdad". Grado de 
114

'Las experiencias son las que entre-

FRASES

DEL DIA

nan el subconsciente

L. - - - - - - -

Es mucho más importante desaprender que aprender (Punset)

Si quieres cambiar el mundo, cámbiate a ti mismo (Mahatma Gandhi)

"Las situaciones más complejas que nos encontramos las solucionamos de manera inconsciente". Galán, 2014)

(Sergio

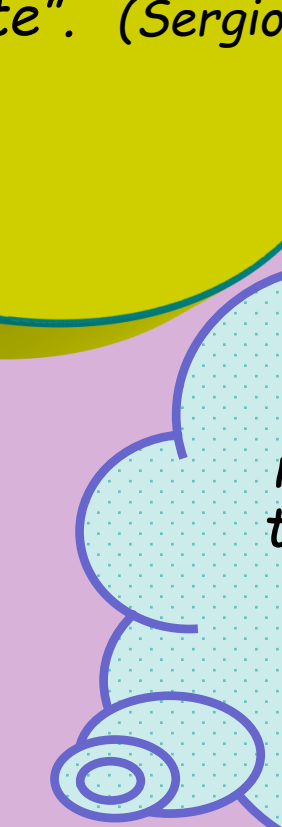

"A menudo, no somos conscientes de las razones y las causas de nuestro propio comportamiento". (Jonh Bargh) to (Joht Bargh) seleccionar la es sel
John bargh)

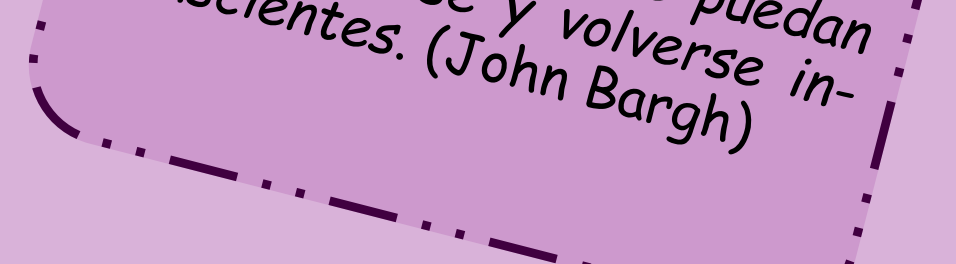

114 


\section{MAPACONCRTILE}

$\bullet \bullet \bullet \bullet \bullet \bullet \bullet \bullet \bullet \bullet \bullet \bullet \bullet \bullet \bullet \bullet \bullet \bullet \bullet \bullet \bullet \bullet \bullet \bullet \bullet \bullet \bullet$

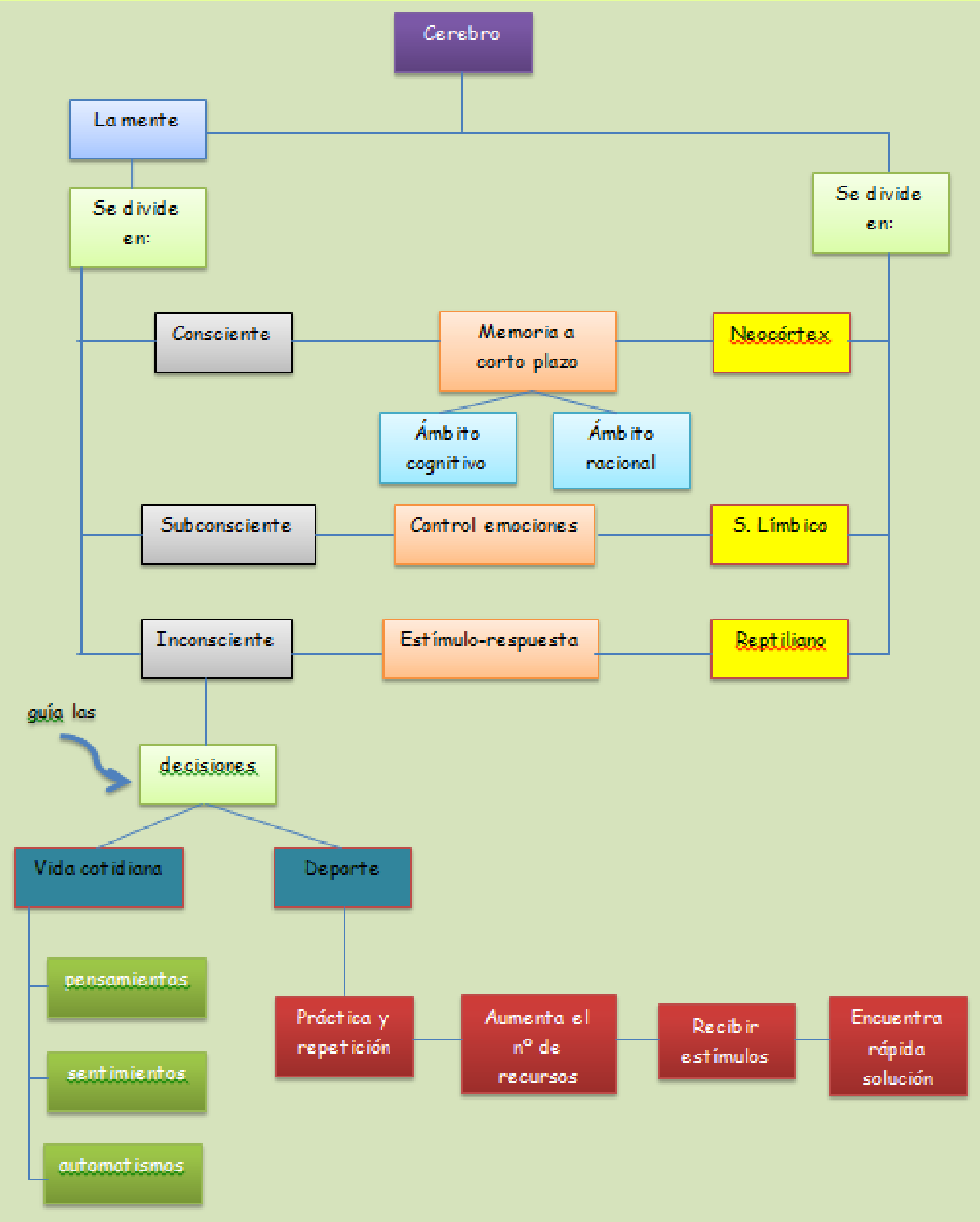

Márquez, M.(2014). Apuntes inéditos de la asignatura "La educación en valores y la práctica de actividad física en colectivos en situación de desigualdad". Grado de Cien- 
¿Cómo se pueden superar nuestros gatillos emocionales? ¿Qué pasaría si nos pusiéramos a pensar en las acciones que nuestro cerebro realiza inconscientemente? ¿Nuestra parte inconsciente es más importante que la consciente?

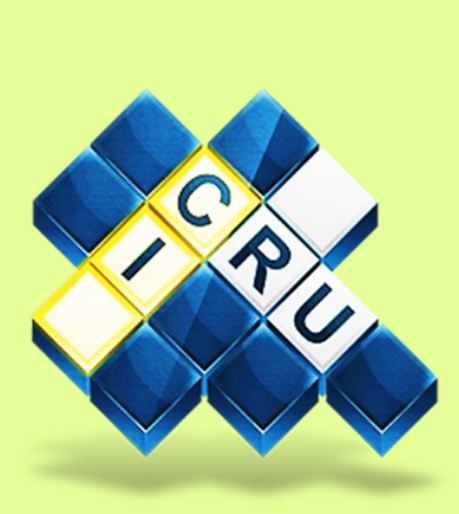

\section{¿En qué partes se divide la mente?}
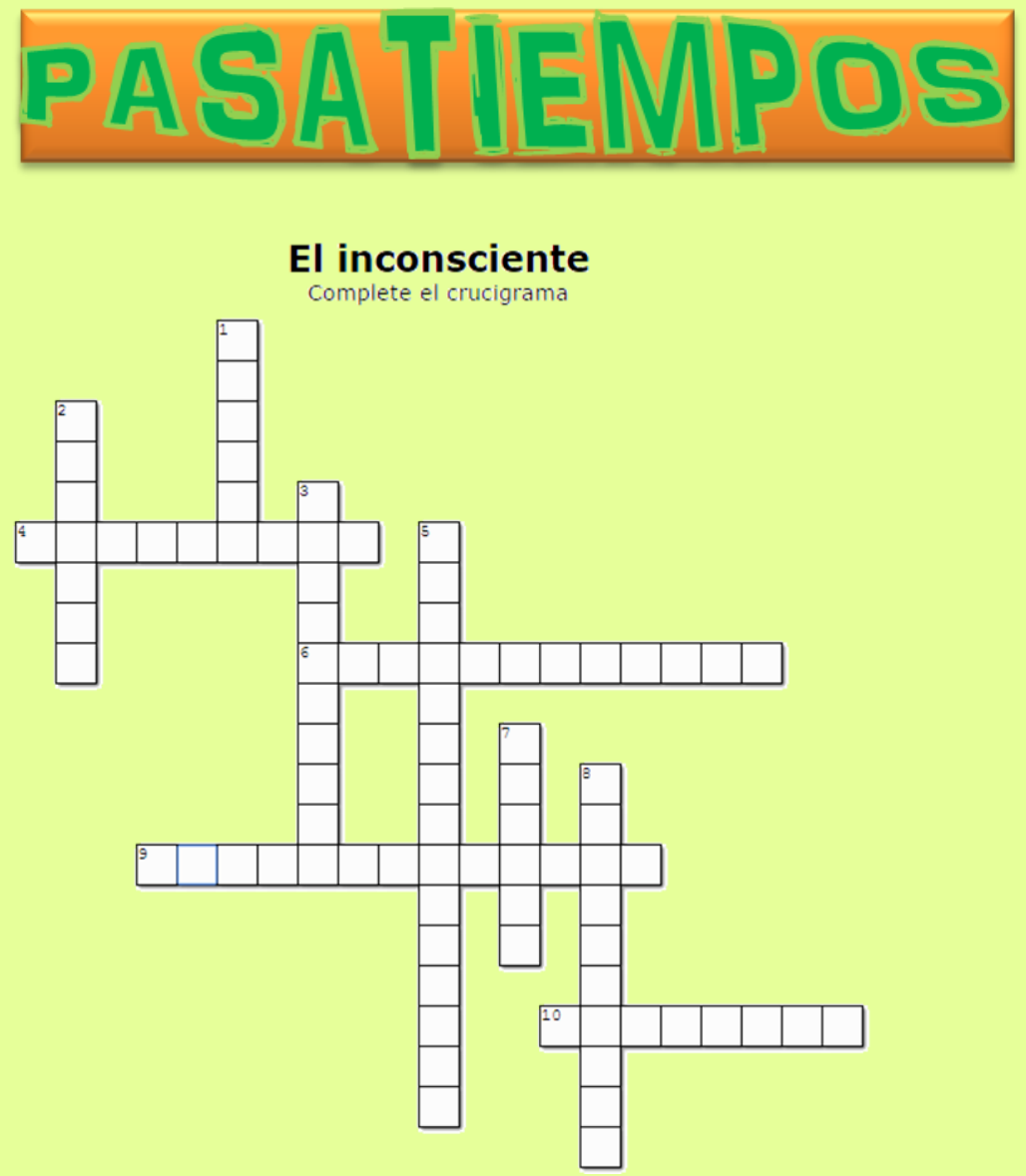

\section{Horizontal}

4. corteza nueva

6. donde se encuentran los instintos

9. referido a lo inconsciente

10. apacidad que tiene alguien para entender las cosas o un objetivo, tenerlo en cuenta o en consideración

\section{Vertical}

1. acción que se realiza en forma de espejo

2. órgano más importante del cuerpo humano

3. parte del cerebro que regula los elementos básicos

5. imagen que tiene cada persona de sí misma

7. imitación de un comportamiento

8. conjunto de percepciones, pensamientos, evaluaciones, sentimientos de uno mismo 


\section{CONCLUSIONES}

- Para superar el miedo hay que ir hasta su raíz y transformarlo en confianza.

- La autoestima es la mejor medicina que hay en este mundo para la resolución de conflictos. A través de la autoestima podemos desarrollar más valores como pueden ser la confianza, la autonomía etc. Se podría decir incluso que la autoestima es el motor que mueve a un equipo mediante el empleo de refuerzos positivos, premiar las buenas actuaciones... por ejemplo ir cambiando el capitán en un equipo para que no sea siempre el mismo y todos sus miembros se sientan útiles y motivados.
- El ejemplo del docente y su actitud es la mayor herramienta por la que podemos desarrollar las emociones.

- La mayoría de las cosas que hacemos en el día a día la hacemos a través del subconsciente y la autoestima es uno de los protagonistas principales para poder actuar correctamente.

- Todos los excesos en la rutina o zona de confort son tapones que tratamos de poner a nuestros agujeros emocionales. La comida, la bebida, el alcohol, la ira, el sexo o la actividad física son tapaderas para nuestros problemas emocionales.

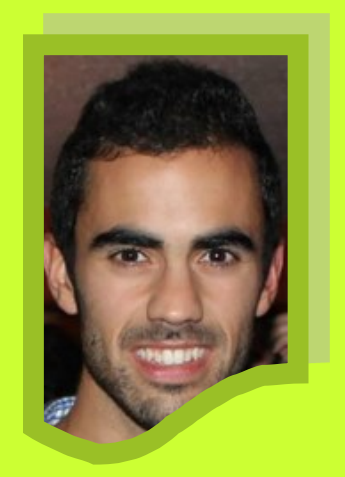

Autor: Álvaro Carrasco

Sáenz-López, P. (2014). Apuntes inéditos de la asignatura "La educación en valores y la práctica de actividad física en

\section{REFEREMIIIIS B|BLIOGRÁFICAS}

\section{-> ARTÍCULOS:}

* Dominguez, A. y Yáñez, J. (2011). El Inconsciente: Una Mirada sobre su Historia y sus Retos Actuales. Psychología Latina, Vol. 2, No. 2, 172-183. Universidad Nacional de Colombia.

* García, H. (2010). James y Freud: convergencias y divergencias alrededor del inconsciente. Revista de historia de la psicología, vol. 31, núm. 2-3 (junio-septiembre) 75-88. Universitat de València. Valencia (España).

* Hospital Sant Joan de Déu de Barcelona (2012). La actividad física mejora el aprendizaje y el rendimiento escolar. Recuperado en http://www.infocop.es/pdf/Faros7Deporte.pdf

* Melcón Álvarez, A. (1991). Educación en la autoestima. Revista complutense de educación. ISSN 11302496, vol. 2, n⿳⺈ 3, 491-500.

* Mora, J. A., Zarco, J. A. y Blanca, M.J. (2001). ATENCIÓN-CONCENTRACIÓN COMO ENTRENAMIENTO PARA LA MEJORA DEL RENDIMIENTO DEPORTIVO EN JUGADORES PROFESIONALES DE FÚTBOL. Revista de Psicología del Deporte, Vol. 10, núm. 1, pp. 49-65. Universitat de les Illes Balears, Universitat Autònoma de Barcelona

* Moreno, J.A. et al. (2006). Importancia de la práctica físico-deportiva y del género en el auto concepto físico de los 9 a los 23 años. 
* Murphy, J. (2009). EL PODER DE TU MENTE SUBCONSCIENTE: USANDO EL PODER DE TU MENTE PUEDES ALCANZAR UNA PROSPERIDAD, UNA FELICIDAD Y UNA PAZ MENTAL SIN LÍMITES. ARKANO BOOKS. HTTP://WWW.CASADELLIBRO.COM/LIBRO-EL-PODERDE-TU-MENTE-SUBCONSCIENTE-USANDO-EL-PODER-DE-TU-MENTE-P-UEDESALCANZAR-UNA-PROSPERIDAD-UNA-FELICIDAD-Y-UNA-PAZ-MENTAL-SINLIMITES/9788496111769/1610083

* Parrado, C. LAS CUATRO LEYES DE LA MENTE SUBCONSCIENTE Y SU APLICACIÓN PARA TRIUNFAR. http://www.bubok.es/libros/187775/LAS-CUATRO-LEYES-DE-LA-MENTESUBCONSCIENTE-Y-SU-APLICACION-PARA-TRIUNFAR

\section{->ENLACES ON.-LINE Y WEBSITES:}

* Nelson, T. (1997). La venganza del inconsciente. http://topicosgerenciales.wordpress.com/2007/12/20/lavenganza-del-inconsciente/

* Nieri, D. El miedo al Éxito. Asociación de Psicología del Deporte Argentina. http://www.psicodeportes.com/articulos/psicologia_deporte/El_miedo_al_exito.pdf

* Vázquez, Piatti, M. (2008). El autoconocimiento, la base de la autoestima. ABC. Recuperado en: http:// www.abc.com.py/articulos/el-autoconocimiento-la-base-de-la-autoestima-1059212.html

* http://www.rtve.es, a 17 de diciembre de 2014:

$\underline{\text { http://www.rtve.es/alacarta/videos/para-todos-la-2/para-todos-2-debate-inconsciente/2351589/ }}$

* http://www.rtve.es, a 17 de diciembre de 2014:

http://www.rtve.es/alacarta/videos/redes/redes-decisiones-son-inconscientes/1016325/

* https://www.youtube.com, a 17 de diciembre de 2014:

https://www.youtube.com/watch?v=t4eaTx7bCew

* https://www.youtube.com, a 17 de diciembre de 2014:

http://www.youtube.com/watch?v=Obq6aZXxBhs

* https://www.youtube.com, a 7 de enero de 2015: http://www.youtube.com/watch?v=TyrlSGUt6cY

* http://mejorconsalud.com, a 8 de enero de 2015: http://mejorconsalud.com/descubre-la-ley-del-espejo-para-ser-feliz/

* http://www.youtube.com, a 11 de diciembre de 2014: ttp://www.youtube.com/watch?v=H9DjChVsTbY

* http://www.youtube.com, a 12 de diciembre de 2014: https://www.youtube.com/watch?v=4tYu6VcJ0oU

* http://www.youtube.com, a 12 de diciembre de 2014: http://www.youtube.com/watch?v=pla--a3Uzhg

* http://www.youtube.com, a 12 de diciembre de 2014: http://www.youtube.com/watch?v=L5O3DYjZ_IE

*Sergio Galán , 2014. Blog de Diego Umaña. Consultado en: http://africandiego.blogspot.com.es/2014_11_01_archive.html 